\title{
Ailuropoda melanoleuca
}

What animal is black and white and loved all over the world? If you guessed the giant panda, you're right! The giant panda is also known as the panda bear or bamboo bear, or in Chinese as Daxiongmao, the "large bear cat." In fact, its scientific name means "black and white cat-footed animal."

Giant pandas are found only in the mountains of central China-in small isolated areas of the north and central portions of the Sichuan Province, in the mountains bordering the southernmost part of Gansu Province and in the Qinling Mountains of the Shaanxi Province.

Giant pandas live in dense bamboo and coniferous forests at altitudes of 5,000 to 10,000 feet. The mountains are shrouded in heavy clouds with torrential rains or dense mist throughout the year.

Giant pandas have existed since the Pleistocene Era (about 600,000 years ago), when their geographic range extended throughout southern China. Fossil remains also have been found in present-day Burma.

Giant pandas are bear-like in shape with striking black and white markings. The ears, eye patches, legs and shoulder band are black; the rest of the body is whitish. They have thick, woolly coats to insulate them from the cold. Adults are four to six feet long and may weigh up to 350 pounds - about the same size as the American black bear. However, unlike the black bear, giant pandas do not hibernate and cannot walk on their hind legs.

The giant panda has unique front pawsone of the wrist bones is enlarged and elongated and is used like a thumb, enabling the giant panda to grasp stalks of bamboo. They also have very powerful jaws and teeth to crush bamboo. While bamboo stalks and roots make up about 95 percent of its diet, the giant panda also feeds on gentians, irises, crocuses, fish, and occasionally small rodents. It must eat 20 to 40 pounds of food each day to survive, and spends ten to 16 hours a day feeding.

The giant panda reaches breeding maturity between four and ten years of age. Mating usually takes place in the spring, and three to five months later, one or two cubs weighing three to five ounces each are born in a sheltered den. Usually only one cub survives. The eyes open at $1 \frac{1}{2} 2$ to two months and the cub becomes mobile at approximately three months of age. At twelve months the cub becomes totally independent. While their average life span in the wild is about fifteen years, giant pandas in captivity have been known to live well into their twenties.

Scientists have debated for more than a century whether giant pandas belong to the bear family, the raccoon family or a separate family of their own. This is because the giant panda and its cousin, the lesser or red panda, share many characteristics with both bears and raccoons. Recent DNA analysis indicates that giant pandas are more closely related to bears and red pandas are more closely related to raccoons. Accordingly, giant pandas are categorized in the bear family while red pandas are categorized in the raccoon family.

In 1869, a French missionary and naturalist named Pere Armand David was the first European to describe the giant panda. In 1936, clothing designer Ruth Harkness

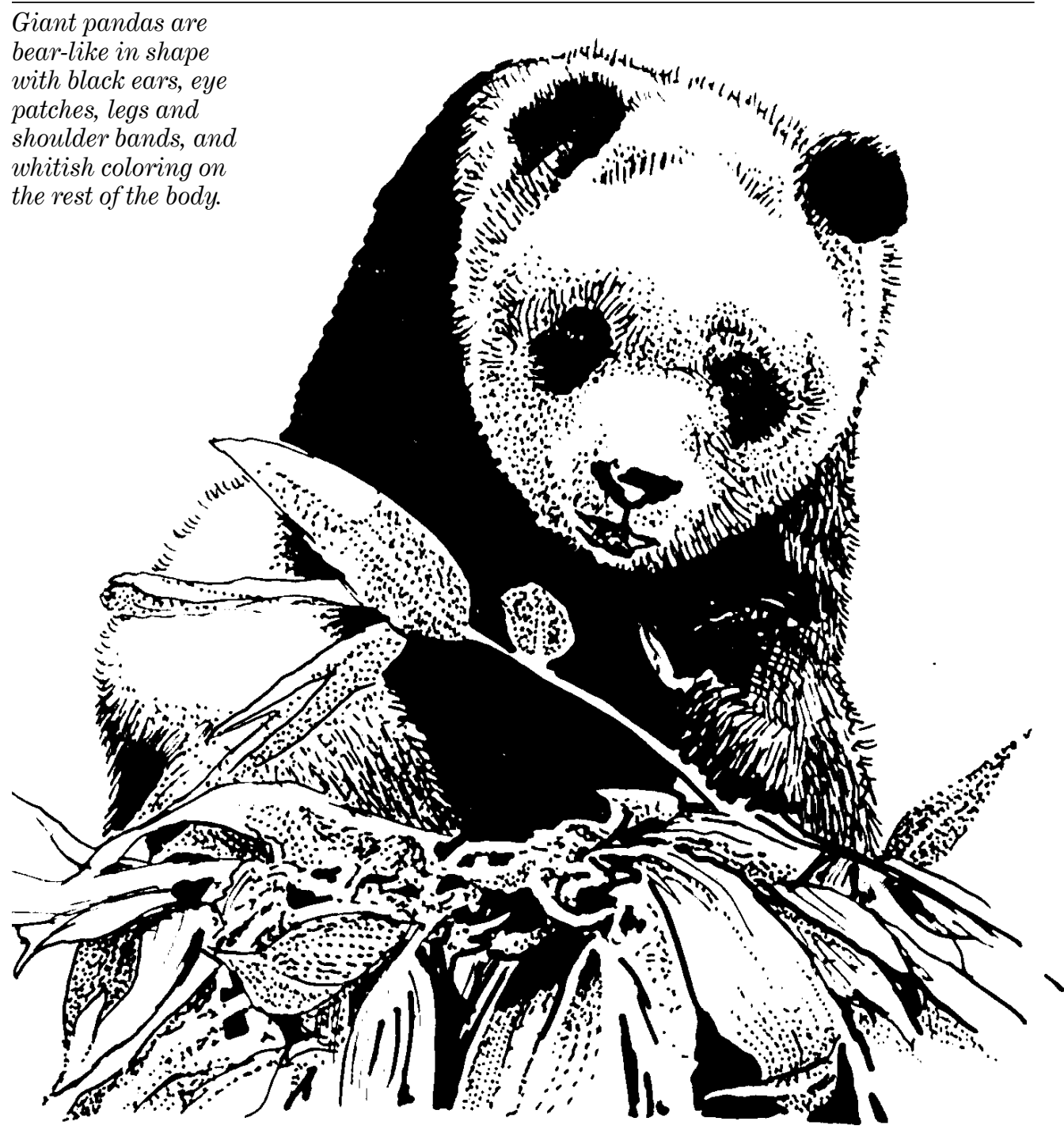


brought the first live giant panda, named Su-Lin, out of China and to the West. Su-Lin lived at Chicago's Brookfield Zoo and was a celebrity until he died in 1938. Today, 124 giant pandas are found in Chinese zoos. Only about 20 giant pandas live in zoos outside of China. In 1980, the first giant panda birth outside China occurred at the Mexico City Zoo.

Until recently, Washington, D.C.'s National Zoo housed Ling-Ling and Hsing-Hsing, perhaps the most well-known giant pandas in North America. A gift from the People's Republic of China to the people of the United States, they were presented as a gesture of amity and goodwill to President Richard Nixon when he visited China in 1972. Ling-Ling, at age 23, died in December 1992.

Giant pandas are among the rarest mammals in the world - there are probably fewer than 1,000 left in the wild. Although adult giant pandas have few natural enemies, the young are sometimes preyed upon by leopards.

Habitat encroachment and destruction are the greatest threats to the continued existence of the giant panda. This is mainly because of the demand for land and natural resources by China's 1 billion inhabitants. To offset this situation, the Chinese government has set aside eleven nature preserves where bamboo flourishes and giant pandas are known to live.

Giant pandas are also susceptible to poaching, or illegal killing, as their dense fur carries a high price in illegal markets in the Far East. The Chinese government has imposed severe penalties for those convicted of poaching giant pandas.

The low reproductive capacity of the giant panda makes it more vulnerable to these threats and less capable of rebounding from its low numbers.

In 1984 the U.S. Fish and Wildlife Service listed the giant panda as an endangered species under the Endangered Species Act of 1973. Endangered means a species is considered in danger of extinction throughout all or a significant portion of its range. The less dire designation of threatened means that a species is likely to become endangered in the foreseeable future. This protection also prohibits giant pandas from being imported into the United States except under certain conditions.

The giant panda is also protected under the Convention on International Trade in Endangered Species of Wild Fauna and Flora (CITES), a treaty that monitors and controls international trade in
Giant pandas eat 20 to 40 pounds of food each day, mostly bamboo, and spend ten to 16 hours a day feeding.

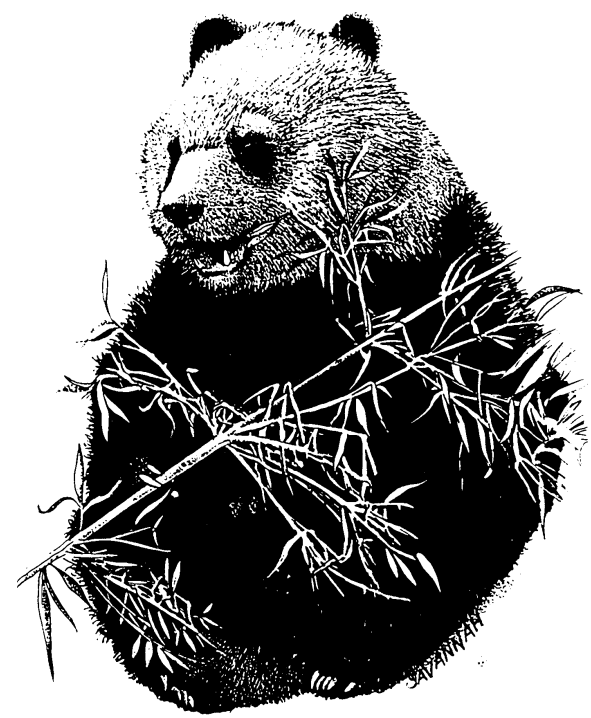

endangered animal and plant species. The Fish and Wildlife Service is the federal agency responsible for the U.S. government's implementation of CITES.

The Service has established a panda policy to inform the public about what is required to qualify for giant panda importation permits. The policy focuses on the conservation of the species in the wild.
The giant panda has unique front paws. One of the wrist bones is enlarged and elongated and is used like a thumb, enabling the panda to grasp bamboo stalks.
Fewer than 1,000 giant pandas remain in the wild, making it one of the world's rarest mammals. Habitat encroachment and destruction as well as illegal killing are the main causes of the giant panda's decline.

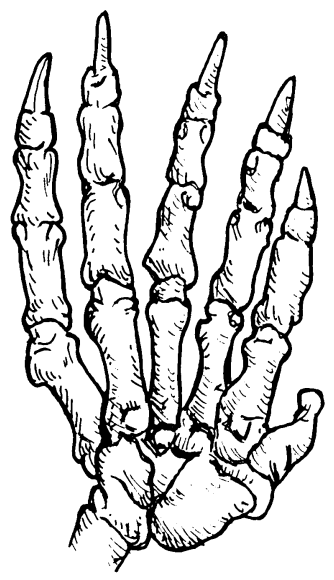

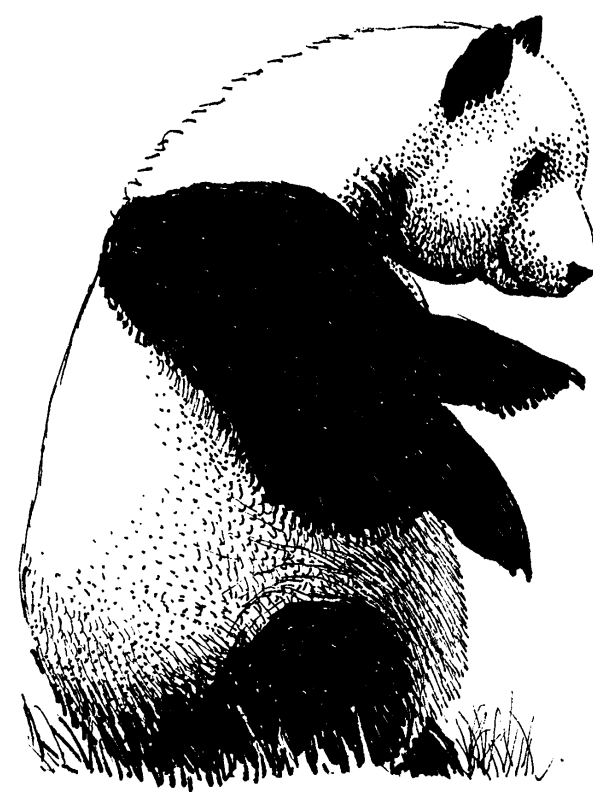

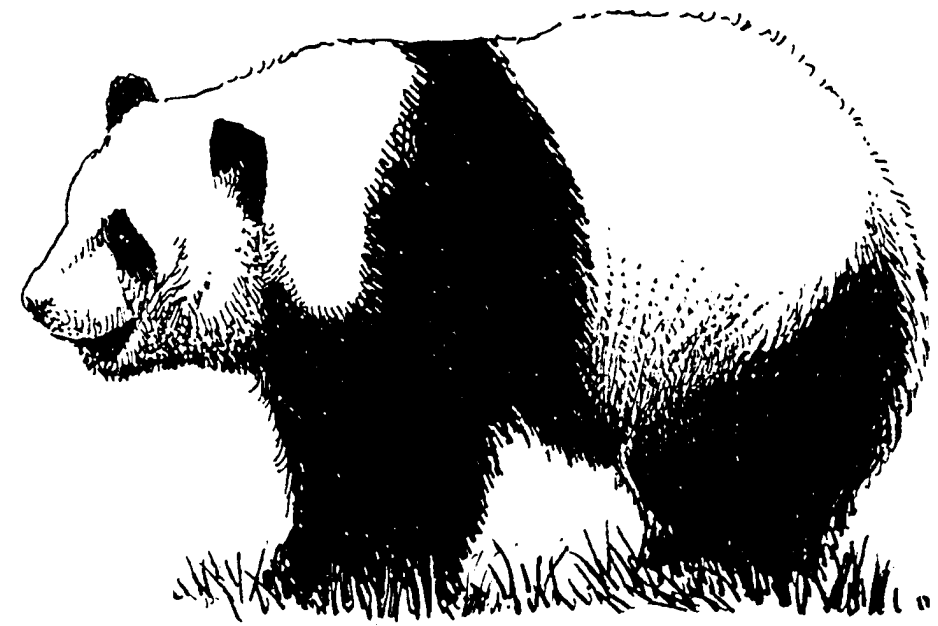

\author{
U.S. Fish \& Wildlife Service \\ $1800 / 344$ WILD \\ http://www.fws.gov \\ June 1999
}

